\title{
ASOCIACIÓN DE LA PARTICIÓN HEPÁTICA Y LIGADURA DE LA VENA PORTAL PARA HEPATECTOMÍA POR ETAPAS, UN NUEVO PROCEDIMIENTO EN CIRUGÍA HEPÁTICA*
}

\author{
Drs. Mario Uribe M. ${ }^{1,2,3}$, Sebastián Uribe-Echevarría A. ${ }^{1}$, Julio Núñez V. ${ }^{2}$, \\ Waldo Martínez M. ${ }^{1}$, Luis Paqui M. ${ }^{2}$, Juan Pablo Camacho M. ${ }^{2}$, Pamela Romanque U. ${ }^{2}$ \\ Servicio de Cirugía. \\ 2 Departamento de Cirugía Oriente, Facultad de Medicina, Universidad de Chile. \\ Hospital del Salvador, Santiago. Chile.
}

\begin{abstract}
Associating liver partition and portal vein ligation for staged hepatectomy, a new hepatic procedure
\end{abstract}

Introduction: Hepatic resection is the standard treatment for the liver tumors and in most cases the only potentially curative option. The most feared complication, in the major hepatic resection, is the postoperative liver failure. A new technique has recently been published in the literature: Liver partition associated to a portal vein ligation for staged hepatectomy. This technique allows an accelerated hipertrophy of the remnant liver, facilitates greater resection surgery, reducing the risk of postoperative hepatic ischemia. The results reported in the literature are promising. We report the first case in Chile with its evolution to 2 years and a discussion about the topic.

Key words: Liver neoplasms, liver regeneration, liver failure, hepatectomy, portal vein ligation.

\section{Resumen}

La resección hepática es el tratamiento estándar para los tumores hepáticos malignos y en la mayoría de los casos la única opción potencialmente curativa. La complicación más temida, en las grandes resecciones, es la insuficiencia hepática postquirúrgica. Una nueva técnica ha sido recientemente publicada en la literatura: La partición hepática asociada a la ligadura de la vena portal para realizar una hepatectomía por etapas. Esta técnica permite una hipertrofia acelerada del hígado remanente que posibilita una cirugía resectiva mayor, disminuyendo el riesgo de isquemia hepática postquirúrgica. Los resultados expuestos en la literatura son promisorios. Presentamos el primer caso clínico en Chile con su evolución a 2 años y una discusión del tema.

Palabras clave: Neoplasias hepáticas, regeneración hepática, falla hepática, hepatectomía, ligadura vena portal.

*Recibido el 17 de junio de 2014 y aceptado para publicación el 12 de agosto de 2014.

Proyecto Fondecyt No 1130254: "New technique to resect liver metastasis with insufficient future liver remnant volume: Split in vivo in situ with portal vein ligation. Mechanisms involved in liver volume gaining: shear stress or metabolic signaling?"

No existe conflicto de interés entre los autores del artículo, el contenido del artículo y alguna empresa en salud.

Correspondencia: Dr. Mario Uribe M.

muribemd@hotmail.com 


\section{Introducción}

En pacientes con tumores hepáticos malignos primarios o secundarios, la única opción potencialmente curativa es la resección hepática ${ }^{1}$. A lo anterior se le han asociado importantes factores limitantes. Uno de ellos es el manejo perioperatorio que ha mejorado en las últimas 2 décadas, incluso hepatectomías mayores se pueden realizar con morbi-mortalidad aceptables ${ }^{2}$. Otro es la experiencia del cirujano que interviene, lo que se soluciona teniendo centros de referencia que concentren la experiencia necesaria para disminuir la tasa de complicaciones ${ }^{2}$. Por último, un factor decisivo es el "remanente hepático futuro" (RHF), que en casos de función hepática normal se sugiere dejar un porcentaje no menor de $25 \%$ del peso del hígado, y en casos de disfunción hepática un $40 \%{ }^{3}$.

Durante las últimas 3 décadas uno de los principales desafíos de la cirugía hepatobiliar ha sido la investigación clínica y experimental orientada a evitar o disminuir la insuficiencia hepática post-operatoria ${ }^{1,4}$. Un gran paso en este sentido fue el aporte realizado por Makuuchi, quien en 1980 introdujo el concepto de la embolización de la rama portal derecha, produciendo una hipertrofia del segmento contralateral ${ }^{5}$. Pese a los avances en las posteriores décadas, muchos pacientes presentaban progresión de enfermedad esperando la hipertrofia del RHF ${ }^{1,6}$.

Un trabajo publicado por Schnitzbauer ${ }^{3}$ promete ser el próximo aporte a la cirugía hepatobiliar, realizando en un primer tiempo la ligadura de vena porta derecha asociado a una transección hepática in situ, y en un segundo tiempo completando la hepatectomía derecha ${ }^{3}$. De esta manera se ha comprobado una reducción en los tiempos de hipertrofia del remanente hepático futuro, y una mayor hipertrofia de este último, en comparación a métodos previos ${ }^{6}$.

A continuación se describe el primer caso clínico reportado en Chile.

\section{Caso clínico}

Paciente de sexo femenino, de 44 años de edad, sin antecedentes mórbidos de importancia, presentó en marzo de 2011 un cuadro de obstrucción intestinal. La tomografía axial computada (TC), evidenció una estenosis del colon sigmoides de aspecto neoplásico, y 2 lesiones hepáticas; una en los segmentos VI y VII de 7,6 x 9 centímetros, la otra de 4,7 x 2,8 centímetros en el segmento $\mathrm{V}$, sin lesiones pulmonares.

Se realizó una colostomía laparoscópica, con evolución favorable. El estudio posterior demostró un adenocarcinoma bien a moderadamente diferenciado.
En abril de 2011, la paciente inició quimioterapia neoadyuvante. Recibió 4 ciclos con esquema FOLFOX, el cual se suspendió por disfagia, interpretándose como reacción adversa al Oxaliplatino. La disfagia remitió en forma espontánea y se objetivó la normalidad del tubo digestivo.

Una TC de julio de 2011demostró un volumen de RHF de $280 \mathrm{cc}$.

En agosto de 2011 se realizó una intervención quirúrgica, en la que se evidenciaron múltiples metástasis en hígado derecho y un nódulo en segmento III de $5 \mathrm{~mm}$, el cual se resecó. Se identificaron los elementos del pedículo portal y se ligó la rama derecha de vena porta. El doppler intraoperatorio confirmó ausencia de flujo. La paciente evolucionó en forma favorable.

Se realizó una TC de control 2 meses posterior a la intervención, que no demostró hipertrofia del segmento lateral izquierdo, por lo que se decidió re-intervenir en octubre del mismo año.

Primer tiempo quirúrgico:

Se realizó una incisión subcostal bilateral. Liberación hepática con control vascular. Se ligó la rama portal derecha y se alcoholizó con $10 \mathrm{cc}$ de alcohol absoluto. Se realizó una transección del parénquima hepático in situ, siguiendo la línea delimitada por la isquemia. Las pérdidas hemáticas estimadas fueron de $1.000 \mathrm{cc}$ (Figura 1).

La paciente evolucionó en forma favorable, manejada en la Unidad de Cuidados Intensivos (UCI), fue extubada al segundo día postoperatorio. Durante las primeras $24 \mathrm{~h}$ presentó requerimientos de drogas vasoactivas (DVA) y una elevación de las pruebas hepáticas (Figura 2). En la TC del noveno día postoperatorio se evidenció un aumento del volumen del RHF a $500 \mathrm{cc}$, es decir, un incremento de $220 \mathrm{cc}$, lo que representa un $79 \%$.

Segundo tiempo quirúrgico (14 días posterior a primera intervención):

Se realizó una re-laparotomía, destacándose una gran hipertrofia de lóbulo hepático izquierdo, concordante con los hallazgos de TC, y un tumor móvil de 4 centímetros de diámetro mayor en sigmoides, sin signos de carcinomatosis peritoneal. Se realizó una adherenciolisis y separación de los lóbulos hepáticos previamente seccionados. Se completó la hepatectomía derecha preservando la vena cava inferior retrohepática. Una ecografía doppler intraoperatoria mostró la tríada portal indemne del lóbulo izquierdo remanente. En el mismo acto quirúrgico, se resecó el tumor primario con una operación de Hartmann. Se estimó un sangrado de 1.600 cc (Figura 1).

Realizó su postoperatorio en UCI, sin requerimientos de DVA, ventilando espontáneamente. Evolucionó con alza autolimitada de las pruebas 
1

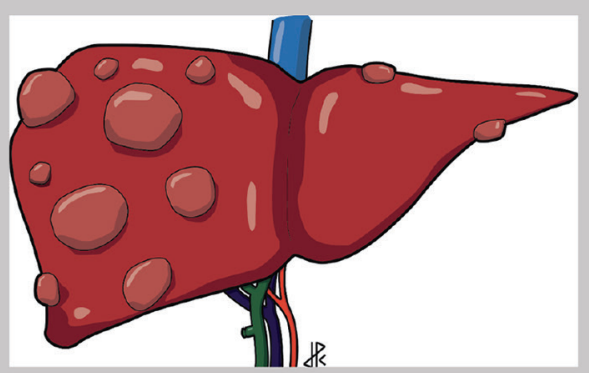

3

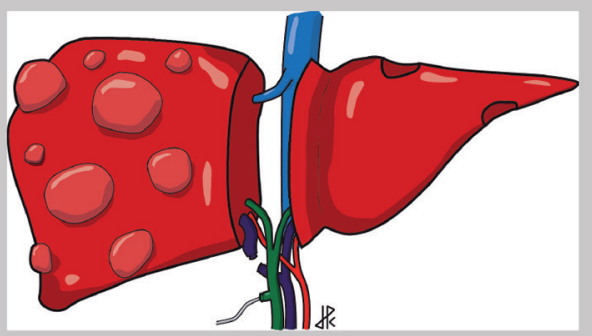

2

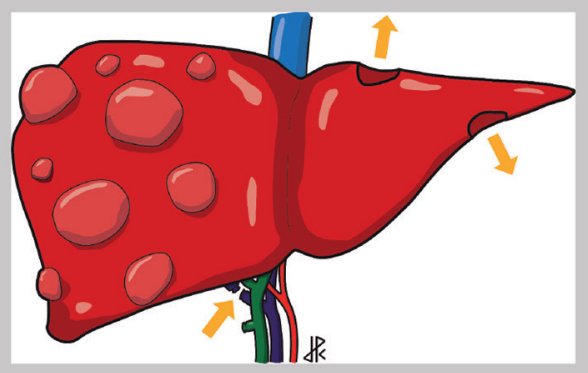

4

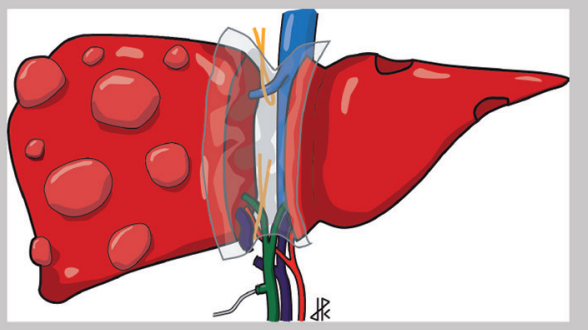

5

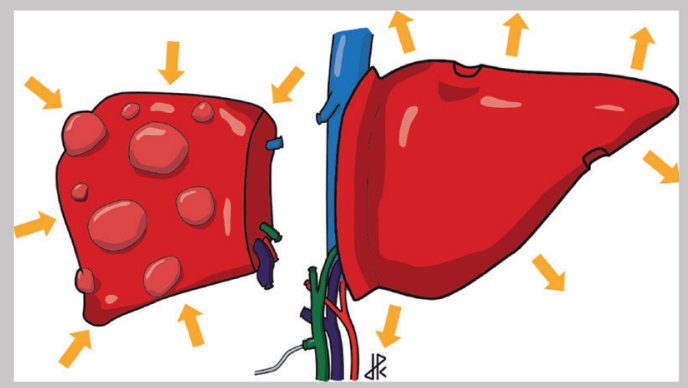

Figura 1. 1) Hígado con metástasis en ambos lóbulos y remanente hepático insuficiente; 2) Primera cirugía: resección de metástasis del segmento lateral izquierdo ("clearence”); 3) Primera cirugía: ligadura o sección rama portal derecha y transección del parénquima; 4) Primera cirugía se deja Sepra Film para disminuir adherencias; 5) Segunda cirugía: se completa la hepatatectomía derecha, una vez confirmado un remanente hepático.

hepáticas durante las primeras $24 \mathrm{~h}$ (Figura 2), y posteriormente presentó una colección intra-abdominal la cual se trató en forma exitosa con drenaje y tratamiento antibiótico según cultivos. Fue dada de alta luego de 27 días posterior a la segunda cirugía.

Recibió 12 ciclos con el esquema FOLFIRI, sin evidencias de recidiva. En octubre de 2012, se realizó la reconstitución de tránsito. El seguimiento continuó en el equipo de coloproctología y oncología médica.

\section{Discusión}

La resección hepática es el tratamiento de elección en la mayoría de los tumores hepáticos que tienen intención curativa ${ }^{1,3,4}$. Sin embargo, en tumores como metástasis hepáticas por carcinoma colorrectal, esto sólo es posible en el $10 \%$ a $20 \%$ de los casos ${ }^{4}$.

Durante las últimas décadas se han desarrollado diferentes técnicas para aumentar la resecabilidad de 


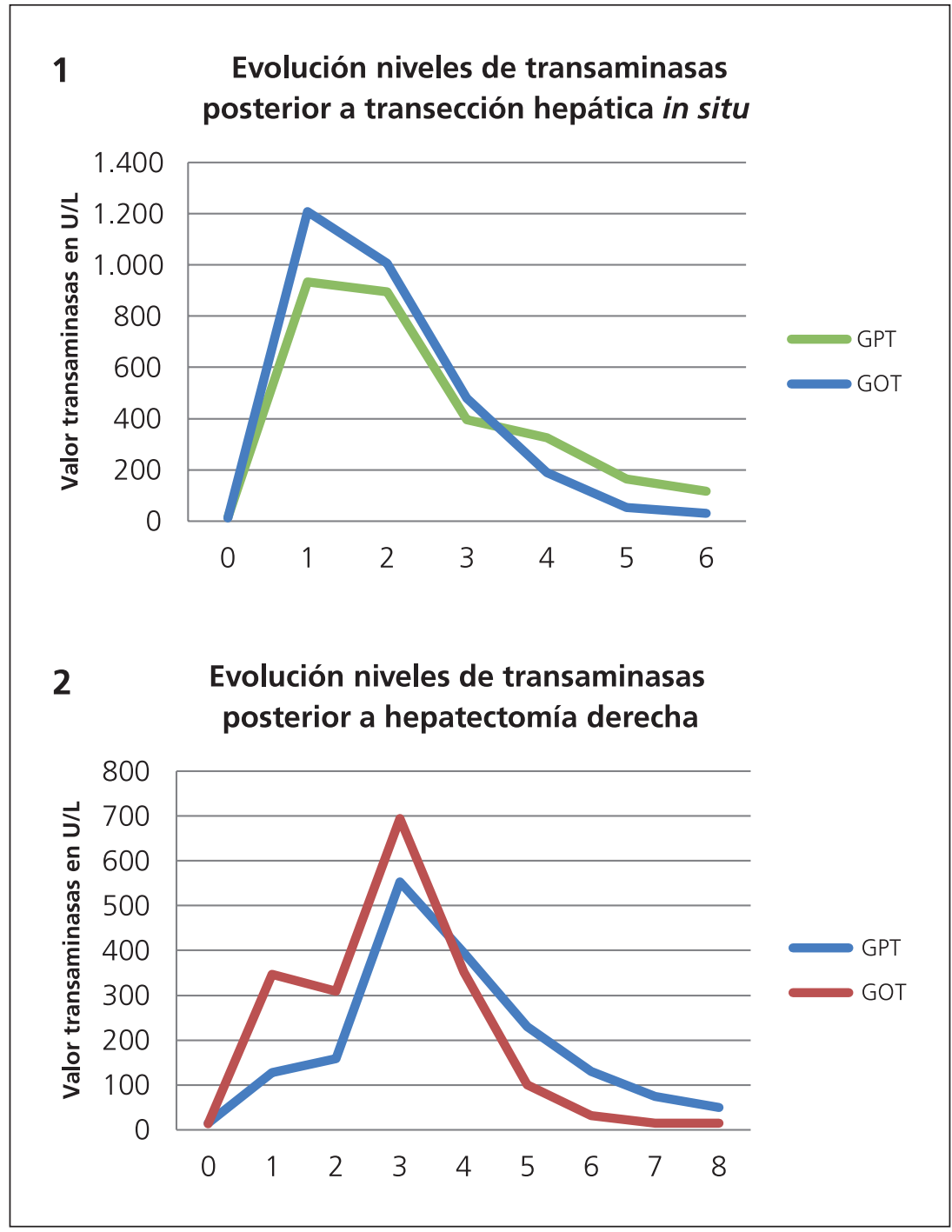

Figura 2. 1) Curva de pruebas hepáticas luego de primera intervención; 2) Curva de pruebas hepáticas luego de segunda intervención.

los tumores hepáticos. Esto incluye oclusión de vena porta $^{5,7,8}$, quimioterapia neoadyuvante arterial ${ }^{9,10}$, inmuno-quimio terapia transarterial ${ }^{11}, \mathrm{y}$ destrucción tumoral local ${ }^{12,13}$.

Pese al desarrollo de las nuevas terapias, uno de los factores limitantes ha sido el RHF, particularmente en paciente con compromiso hepático bilateral. En el RHF no sólo es el volumen de éste el que determinará la factibilidad técnica y el riesgo de falla hepática postoperatoria, sino que también son las características del parénquima hepático, tales como presencia de esteatosis, hepatitis, cirrosis, colestasia, tiempo de isquemia, e hígado sometido a quimioterapia ${ }^{14}$. De esta manera la falla hepática producto de un volumen hepático residual funcional insuficiente se presenta como la principal causa de muerte postoperatoria ${ }^{4}$.

Uno de los avances más relevantes en dirección a resolver esta problemática fue el planteamiento de la cirugía en 2 tiempos, la que inicialmente consistió en limpiar un lóbulo hepático mediante métodos resectivos y/o ablativos, seguido de una recuperación de 4 a 6 semanas. De esta manera se espera una hipertrofia del lóbulo intervenido, para realizar posteriormente la lobectomía del lado contralateral, la cual presenta la mayor carga tumoral ${ }^{3}$.

A continuación al concepto anterior se agregan 2 técnicas, la embolización portal y la ligadura de la vena porta, asociado o no a procedimientos para reducir la carga tumoral, logrando incrementar lo 
que será finalmente el volumen del RHF, y a su vez atrofiar el lóbulo contralateral. Estudios recientes han demostrado que la oclusión portal logra un aumento del RHF entre un $10 \%$ a $46 \%$, en un período de 2 a 8 semanas. De esta manera se reducen los tiempos entre una intervención y la siguiente $\mathrm{e}^{1,4,5,15,16}$.

Los anteriores métodos han logrado disminuir la posibilidad de una eventual falla hepática, y volver resecables tumores que inicialmente no lo eran, pero persistía la necesidad de disminuir el tiempo entre la primera intervención y la siguiente, ya que existían pacientes en los que la enfermedad progresaba, haciendo inviable nuevas intervenciones ${ }^{1,4,5,15,16}$.

Recientemente fue publicado un artículo de Schnitzbauer ${ }^{3}$, en marzo de 2012, una hepatectomía derecha extendida en 2 etapas. Inicialmente se realiza una exploración quirúrgica, ligadura de la rama portal derecha, y transección hepática in situ, a lo largo del lado derecho del ligamento falciforme, produciendo una rápida hipertrofia del lóbulo izquierdo, en pacientes con tumores hepáticos marginalmente resecables o irresecables en un principio, ya sean primarios o secundarios. En un segundo tiempo se completa la hepatectomía derecha. Luego de una mediana de intervalo de 9 días, la mediana de volumen del lóbulo izquierdo fue de $536 \mathrm{cc}$, con un delta de volumen de $225 \mathrm{cc}$, y un incremento de volumen de $74 \%{ }^{3}$. Numerosos artículos y comentarios han sido posteriormente publicados al respecto ${ }^{6,16-21}$.

El caso presentado es el primero realizado y publicado en Chile con esta técnica, además de ser uno de los primeros en el mundo de tener seguimiento a largo plazo (2 años), con sobrevida libre de enfermedad hepática hasta la actualidad. En este caso el rol de la bipartición es evidente, ya que no se produjo hipertrofia del lóbulo izquierdo cuando sólo se ligó la vena porta derecha. Los resultados obtenidos a corto plazo en nuestra paciente son similares a los reportados en la literatura existente t,16-21. $^{\text {. }}$

Si bien falta aún mayor estudio en sus aplicaciones y seguimiento a largo plazo, para poder definir con mejor precisión sus indicaciones, muchos centros quirúrgicos internacionales han incorporado esta técnica en su arsenal terapéutico.

\section{Referencias}

1. Clavien PA, Petrowsky H, DeOliveira M, Graf R. Strategies for Safer Liver Surgery and Partial Liver Transplantation N Engl J Med. 2007;356:1545-59.

2. Rando K, Harguindeguy M, Leites A, Ettlin A, González $\mathrm{S}$, Scalone P, y cols. Indicadores de calidad en cirugía hepática: influencia del trabajo en equipo multidisciplinario y del "efecto centro". Acta Gastroenterológica Latinoamericana 2010;40:10-21.
3. Schnitzbauer A, Lang S, Goessmann H, Nadalin S, Baumgart J, Farkas S, et al. Right portal vein ligation combined with in situ splitting induces rapid left lateral Liver lobe hypertrophy enabling 2-Staged extended right hepatic resection in small-for-size settings. Ann Surg. 2012;255:405-14.

4. Jaeck D, Oussoultzoglou E, Rosso E, Greget M, Weber JC, Bachellier P. A Two-Stage Hepatectomy Procedure Combined With Portal Vein Embolization to Achieve Curative Resection for Initially Unresectable Multiple and Bilobar Colorectal Liver Metastases. Ann Surg. 2004;240:1037-49; discussion 1049-51.

5. Makuuchi M, Thai BL, Takayasu K, Takayama T, Kosuge T, Gunvén P, et al. Preoperative portal embolization to increase safety of major hepatectomy for hilar bile duct carcinoma: a preliminary report. Surgery 1990;107:521-7.

6. Santibañes E, Clavien PA. Playing Play-Doh to Prevent Postoperative Liver Failure The "ALPPS" approach Ann Surg. 2012;255:415-7.

7. Azoulay D, Castaing D, Smail A, Adam R, Cailliez V, Laurent A, et al. Resection of non resectable liver metastases from colorectal cancer after percutaneous PVE. Ann Surg. 2000;231:480-6.

8. Elias D, Roche A, Vavasseur D, Lasser P. Induction of hypertrophy of a small left hepatic lobe by preoperative right portal embolization, preceding extended right hepatectomy. Ann Chir. 1992;46:404-10.

9. Bismuth H, Adam R, Lévi F, Farabos C, Waechter F, Castaing D, et al. Resection of nonresectable liver metastases from colorectal cancer after neoadjuvant chemotherapy. Ann Surg. 1996;224:509-22.

10. Miyanari N, Mori T, Takahashi K, Yasuno M. Evaluation of aggressively treated patients with unresectable multiple liver metastases from colo- rectal cancer. Dis Colon Rectum 2002;45:1503-9.

11. Lygidakis NJ, Vlachos L, Raptis S, Rassidakis G, Balaskas C, Sgourakis G, et al. New frontiers in liver surgery: two-stage liver surgery for the management of advanced metastatic liver disease. Hepato-Gastroenterology 1999;46:2216-28.

12. Adam R, Akpinar E, Johann M, Kunstlinger F, Majno $\mathrm{P}$, Bismuth H. Place of cryosurgery in the treatment of malignant liver tumors. Ann Surg. 1997;225:39-50.

13. Curley SA, Izzo F, Delrio P, Ellis LM, Granchi J, Vallone $\mathrm{P}$, et al. Radiofrequency ablation of unresectable primary metastatic hepatic malignancy: results in 123 patients. Ann Surg. 1999;230:1-8.

14. Clavien PA, Oberkofler CE, Raptis DA, Lehmann K, Rickenbacher A, El-Badry AM. What Is Critical for Liver Surgery and Partial Liver Transplantation: Size or Quality? Hepatology 2010;52:715-29.

15. Adam R, Laurent A, Azoulay D, Castaing D, Bismuth H. Two-Stage Hepatectomy: A Planned Strategy to Treat Irresectable Liver Tumors. Ann Surg. 2000;232:777-85.

16. Álvarez F, Ardiles V, Sánchez R, Pekolj J, Santibañes 
E. Associating Liver Partition and Portal Vein Ligation for Staged Hepatectomy (ALPPS): J Gastrointest Surg. 2013;17:814-21.

17. O Martins O, Assunção JM, Lima N, Moura A. Associating Liver Partition and Portal Vein Ligation for Staged Hepatectomy (ALPPS): A New Approach in Liver Resections. Arq Bras Cir Dig. 2012;25:290-2.

18. Govil S. Rapid improvement in liver volume induced by portal vein ligation and staged hepatectomy: the ALPPS procedure. HPB 2012;14:874.

19. Cavaness K, Doyle MB, Lin Y, Maynard E, Chapman
W. Using ALPPS to Induce Rapid Liver Hypertrophy in a Patient with Hepatic Fibrosis and Portal Vein Thrombosis. J Gastrointest Surg. 2013;17:207-12.

20. Knoefel WT, Gabor I, Rehders A, Alexander A, Krausch M, Schulte am Esch J, et al. In situ liver transection with portal vein ligation for rapid growth of the future liver remnant in two-stage liver resection. British Journal of Surgery 2013;100:388-94.

21. Santibañes E, Álvarez F, Ardiles V. How to Avoid Postoperative Liver Failure: A Novel Method. World J Surg. 2012;36:125-8. 